\title{
State of the Art on Flow Kinematics and Particle Orientations during Composite Processing
}

\author{
CHIBA Kunji \\ Faculty of Education, Shiga University, 2-5-1 Hiratsu, Otsu, Shiga 520-0862, Japan
}

Received 18 July 2006; accepted for publication 7 August 2006

\begin{abstract}
The mechanism of orientation of fibers or thin micro-particles in various flows involving the processing of composite materials has not been fully understood although it is much significant to obtain the knowledge of the processing operations of particle reinforced composites as well as to improve the properties of the advanced composites. The objective of this article is to introduce and well understand the evolution of the particle orientation in a suspension flow and flow kinematics induced by suspended particles by means of both literature review and the following our two research work: Numerical simulation of flow kinematics and fiber orientation for fiber suspension: Orientation of disk-like particles in a micro-composite processing.
\end{abstract}

Key Words: Fiber suspension, Thin particle suspension, Particle orientation evolution, Flow kinematics of suspension, Composite processing

\section{Introduction}

Fiber orientation strongly affects the physical properties of short-fiber reinforced composites and the characterization of fiber orientation distribution becomes much more significant in current processing of high quality composite materials. Fiber suspension can often exhibit anisotropy due to flowinduced fiber alignment. The addition of short fibers to a Newtonian liquid can, therefore, drastically change the flow kinematics even at very low concentrations; Lipscomb et al. [1]; Chiba et al. [2]. Thus the importance of the coupling between flow kinematics and fiber orientation has been recognized during the last two decades [1-10]. Furthermore, an orientation evolution model and a rheological constitutive equation that relates the stress in fiber suspensions to the orientation state are required and should be solved simultaneously to understand and describe the flow kinematics of fiber suspension.

On the other hand, thin micro-particle suspensions, such as talc and mica suspensions, can also exhibit anisotropy due to flow-induced particle alignment. Furthermore, the characterization of the orientation distribution of thin particles strongly affects the mechanical properties of thin micro-particle composites [11-15] and becomes much more significant in current processing of advanced composite materials: e.g., a sheet of micro-particle reinforced composite, in which thin micro-particles migrate to the central plane in the thickness direction and align parallel to the surface, performs a flexible property as well as high quality of thermal $[12,16,17]$ and electrical shielding [18]. Thus the importance of the coupling flow kinematics with thin particle orientation has also been recognized [19].

The mechanism of orientation of fibers or thin microparticles in various flows involving the processing of composite materials, however, has not been fully understood although it is so much significant to obtain the knowledge of the processing operations of particle reinforced composites. The objective of this article is to introduce and well understand the evolution of the particle orientation in a suspension flow and flow kinematics induced by suspended particles by means of both literature review and our research work. In Sec. 2 we will present the literature review on flow kinematics and fiber orientation in fiber suspension flow. In Sec. 3 we will demonstrate the following our research work: (1) Numerical simulation of flow kinematics and fiber orientation for fiber suspension, (2) Orientation of disk-like particles in a micro-composite processing. Finally, in Sec. 4 we will conclude by discussing our understanding and future work on particle suspension flow.

\section{Literature review on fiber suspension flows}

\section{1 Non-recirculationg fiber suspension flow}

Extensive numerical studies on fiber suspension flows have been devoted to extrusion flow, flow around a sphere, 
squeezing flow, radial flow between parallel disks and mold filling flow.

Papanastasiou and Alexandrou [20] investigated the isothermal extrusion of non-dilute fiber suspension in a Newtonian solvent. They used the Dinh-Armstrong constitutive equation [21] to model the rheology of the suspension. The fiber orientation was computed by solving the orientation distribution function along the selected streamlines of the complex velocity field. Rosenberg et al. [3] studied both planar extrusion and falling-ball rheometry of dilute fiber suspension in a Newtonian fluid. They used the transversely isotropic fluid (TIF) model, first proposed by Ericksen [22]. The fiber orientation was obtained by numerically integrating the evolution equation of the second-order orientation tensor with a closure approximation along the streamlines. Phan-Thien and Graham simulated the squeezing flow between two circular disks of a model suspension [23] and the flow past a sphere [24]. The suspension was assumed to be a dilute suspension of rigid spheroids of large aspect ratio and was adequately modeled by the TIF model. Ranganathan and Advani [5] studied an axisymmetric diverging radial flow between disks of nondilute fiber suspension. They coupled a modified form of the rheological equation given by Shaqfeh and Fredrickson [25] with a model for the evolution of fiber orientation which took account of the effect of fiber-fiber interactions on the fiber angular velocities. Amhed and Alexandrou [6] investigated the filling of a simple two-dimensional injection molding cavity. The constitutive relation proposed by Dinh and Armstrong was used to express the stress tensor. And also the fiber orientation was computed by solving the orientation distribution function along the streamlines. Altan and Rao [7] developed the closed-form solution of the 3-D orientation field induced by a radially diverging, steady Newtonian flow between two parallel disks. Furthermore, the accuracy and performance of the closure approximations used in the orientation evolution equations were investigated. Chung and Kwon [8] predicted the transient behavior of fiber orientations, together with a mold filling simulation of short-fiber suspension in arbitrary 3-D mold cavities. The Dinh-Armstrong model was incorporated into the Hele-Shaw equation and the evolution equation of the second-order orientation tensor was computed to obtain the orientation of fibers.

\section{2 Recirculating fiber suspension flow}

The characterization of the fiber orientation in a recirculating flow is very important from a practical view point because a recirculating flow occurs in a complex flow which is often seen in polymer processing industries. There has been work on fiber suspension flows including a recirculating flow as well as fiber orientation in a recirculating flow.

Lipscomb et al. [1] and Chiba et al. [2] studied the circular entry flow of fiber suspensions in a Newtonian solvent. The TIF model was applied to suspensions with large aspectratio (length/diameter) fibers. The results obtained using this model were compared with the flow visualizations and the growth of the salient corner vortex was predicted with high accuracy over the semi-dilute fiber suspension regime, although the constitutive model was derived for dilute fiber suspensions. In the computations, a fiber was approximated to align along the streamline (co-linear fiber alignment). Lipscomb et al. stated that the continuum theory for dilute fiber suspensions that incorporated the orientation distribution function into the stress equation predicted the flow field accurately and the form of the theory was unchanged when extended to semi-dilute suspensions. Furthermore, Baloch and Webster [9] presented the simulations for dilute to semi-dilute fiber suspension flows in a Newtonian solvent through various contraction and expansion geometries using the constitutive model of Lipscomb et al. [1]. They adopted co-linear and orthogonal fiber alignment (the local fiber orientation is perpendicular to the streamline) conditions and compared the simulated results with the experimental observations.

In a previous study [26], we investigated the 2-D fiber orientation in a main flow as well as in a recirculating flow within a salient corner of a 1:4 backward-facing step channel using both numerical simulations and fiber orientation observations. In the numerical simulations, orientations of a large number of fibers in a fluid element could be evaluated from computation of the Jeffery equation [27] along the streamlines: we called this method "statistical scheme". The statistical scheme made it possible to generate the orientation distribution function by means of a large number of fibers without direct solution of this function, such as solution of the Fokker-Plank equation. In this work, fundamental and important results were obtained: in a recirculating flow, all fibers completely align along the streamlines in steady state (co-linear alignment) for large aspect-ratio fibers, while all fibers in a fluid element can completely align in one direction (complete alignment), however, the preferred angle lies obliquely to the streamlines for small aspect-ratio fibers. The fiber orientation in steady recirculating flows was also reported by Chang et al. [28], Azaiez et al. [29], Poitou et al. [30] and Chinesta et al. [31].

\section{3 Tensorial description of particle orientation}

Akbar and Altan [32] stated the drawback of the evolution 
equation of the orientation tensor: although an orientation distribution was necessary to describe the orientation dependent properties of fiber suspensions completely, direct solution of this function or its construction from the solution of numerous discrete fibers did not gain popularity because of mathematical complexity and computational intensity. Consequently, the implementation of tensorial quantities such as orientation tensors found some acceptance for microstructural characterization in the work of Hinch and Leal [33] on suspension rheology and in more recent work of Doi [34] and Grmela and Carreau [35] on conformation tensor rheological model. Orientation tensors provided considerable advantages for numerical simulations because of their concise description of the orientation state. The major drawback of the orientation tensors was the requirement of a closure equation in order to approximate the higher order tensors which appeared in the orientation evolution equations. Advani and Tucker [36, 37], Altan et al. [38], Cintra and Tucker [39], Chung and Kwon [40] and Parsheh et al. [41] tackled the problems related to closure approximations.

Akbar and Altan [32] also proposed a statistical method which made it feasible to generate the orientation distribution function by considering large numbers of fibers without solving the Fokker-Planck equation in order to overcome the drawback of the orientation tensors and difficulty of solution of the Fokker-Planck equation. Hence, the solution of orientation fields along the streamlines in a complex flow was feasible by considering numerous fibers starting from specified initial orientation. The statistical method was used by Ericsson et al. [42] in computations of the rheology of a discontinuous fiber filled polypropylene in a squeezing flow between parallel plates. It was shown that the statistical method gave an excellent prediction of the behavior of the exact solution in simple flows using only 100 test fibers. Furthermore, Chiba and Nakamura [43] used the statistical method to examine the accuracy of the quadratic closure approximation of the fourth-order orientation tensor.

\section{4 Developing fiber suspension flow through a straight channel}

There have been studies concerning a developing flow of fiber suspensions through a straight channel. Altan et al. [44] assumed that the orientation was planar and used the Dinh-Armstrong model [21] for the rheology of the suspension. The orientation state was obtained by calculation of the evolution equation of the fourth-order orientation tensor with a sixth-order quadratic closure approximation. On the other hand, Chono and Makino [45] used the Dinh-Armstrong model and the evolution equation of an equivalent strain tensor derived by Lipscomb [46]. The calculation of this equation, therefore, replaced the solution of the Fokker-Plank equation for the orientation distribution function. They also compared the exact solutions with the predictions using the evolution equations of the fourth-order and sixth-order orientation tensors with a quadratic closure approximation. In practical situations fibers can align in a 3-D manner (so-called out-of-plane orientation of fibers) even in 2-D flows, such as simple shear flow and parallel plate channel flow. Ausias et al. [47] used the constitutive equation developed by Lipscomb et al. [1] and the evolution equation of the fourth-order orientation tensor with a quadratic closure approximation. They computed an axisymmetric flow through an annular tube die by coupling with 3-D orientation of fibers and showed that the predictions of the fiber orientation were in good agreement with the observations in a 30wt $\%$ glass-fiber reinforced polypropylene tubes. Chiba and Chinesta [48] computed the 2-D flows of fiber suspensions with quasi infinite aspectratio fiber or small aspect-ratio fiber $\left(r_{a}=10\right)$ in a Newtonian fluid through a parallel plate channel by coupling flow kinematics with 3-D fiber-orientation distribution. It is well known that fibers of small aspect-ratio frequently and periodically flip over when fiber-fiber interaction does not exist, and then the flip-over phenomenon has a strong influence on the flow kinematics and fiber orientations. Flipover is a phenomenon of the rapid rotation of a slender particle that occurs when it is aligned in the perpendicular direction to the flow.

The above studies on fiber suspension flows concern with the flow kinematics and fiber orientations for mono-disperse fiber suspensions. In the processing of fiber composites, however, length of suspended fibers is always distributed because of fiber breakage during processing. Thus the analysis of the effect of fiber length distribution on the flow kinematics is necessary to understand the processing for multi-disperse fiber suspensions.

In our recent work [49], the development of flow kinematics and fiber orientation distribution through a parallel plate channel was simulated for multi-disperse suspensions with discretely dispersed aspect ratio fibers, and their predictions were compared with those obtained for mono-disperse and bi-disperse suspensions. For suspensions including small-aspect-ratio fibers, flip-over or oscillation phenomenon of the orientation ellipsoid (schematic illustration of fiber orientation distribution) caused the wavy patterns of the velocity profile and the streamlines as well as the rapid and complex variation of the shear stress and the normal stress difference near the channel wall. 


\section{5 Fiber suspension flow through a complex channel}

We can find the following articles focusing on complex flows of fiber suspension: Kabanemi et al. [50] described a fully 3-D transient finite element method for computing the flow kinematics and fiber orientation through a rectangular channel as well as during the filling process of an end-gated plate. The fiber-fiber interaction was taken into account. The material anisotropy behavior was modeled by using the Doraiswamy-Metzner model [51]. Azaiez et al. [29] computed the flow of rigid fiber suspensions in polymeric liquid through a 4:1 planar contraction using three rheological models: the PTT, the FENE-CR and the Carreau models. The flow-governing equations, the constitutive equation and the evolution equation of fiber orientation with the closure approximations were coupled and simultaneously solved, then the predicted flow patterns and fiber orientation were presented. Zirnsak and Boger [52] carried out experimental observations of the flow patterns in an axisymmetric contraction for rigid rod molecules, and demonstrated that the salient corner vortex sizes predicted and observed for dilute and semi-dilute rigid fiber suspensions were similar to those observed for the semidilute rigid rod xanthan gum solutions. Fan et al. [53] described a robust numerical method for solving general flows of non-dilute fiber suspensions in the framework of the CONNFFESSIT method [54]. The algorithm was tested on the flow past a sphere in a tube, and the numerical results were compared with the experimental data of Milliken et al. [55]. The simulations of fiber suspensions, together with shear-induced fiber migration, were also carried out in a concentrated regime using the same numerical strategy. Finally, Chung and Kwon [56] performed numerical analysis of non-isothermal fountain flow of fiber suspensions in a Newtonian fluid through an axisymmetric 3-D geometry without the Hele-Shaw approximation. The evolution equation for the second-order orientation tensor with a closure approximation was adopted to predict the fiber orientations in a concentrated regime. A center-gated disk geometry was used as a benchmark test problem.

\section{6 Direct simulation of fiber suspension flow}

Another way to study suspension behavior is to make a direct simulation where forces acting on each fiber included in a given volume are computed and used to calculate fiber motion and stress in the suspension. This method has been often used for spheres. It has also been used for non-spherical particles by Fan et al. [53] for rigid particles under simple shear, and by Schmid et al. [57] for non-rigid particles.
Thomasset et al. [58] computed a direct simulation in two dimension including walls. Yamamoto and Matsuoka [59, 60] and Joung et al. [61, 62] made direct simulations where the fibers are modeled by an array of spheres and they predicted the behavior of rigid and flexible fiber suspensions. Yamamoto and Matsuoka [63] used a similar method to compute the dynamics of plate-like particle suspension. Pozrikidis [64] made a direct simulation for concentrated suspensions of spheres and prolate spheroidal particles. In all of these publications on direct simulation, all particles have the same aspect ratio and they all flip over nearly at the same time in the flow, thus producing large periodic fluctuations in stresses in the suspension. Ausias et al. [65] reported a numerical method, which took account of shortrange hydrodynamic interaction and contact forces, in order to simulate fiber suspension in transient and steady shear flows for concentrated solutions. Fibers were assumed to have varying lengths as observed in industrial composites.

\section{Research on flow kinematics and particle orientation}

In the processing of fiber composites length of suspended fibers is always continuously distributed because of fiber breakage during processing [66] except for compression molding. Thus it is necessary to elucidate the effect of continuous distribution of fiber lengths on the flow kinematics. In the work of Chiba and Chinesta [67], coupled flow kinematics and 3-D fiber orientation distribution was simulated for multi-disperse suspensions with continuously distributed aspect-ratio fibers, then the mechanism of the flip-over or oscillation phenomenon of an orientation ellipsoid, schematic orientation state of fibers can be illustrated using the orientation ellipsoid, was examined in detail.

On the other hand, the evolution of the orientation of thin micro-particles, such as talc and mica which were modeled by a thin disk-like particle, in both a planar extensional flow and a simple shear flow of Newtonian fluids through a large reservoir to a slit channel was analyzed by numerical simulation. Furthermore, the orientation observation was carried out using talc suspension, and numerically simulated results were compared with the observations [68].

\section{1 Numerical simulation for fiber suspension flow}

Fiber suspensions that were the focus of the previous work [67] involve rigid fibers in a Newtonian liquid. In spite of the significant impact of fiber-fiber and fiber-wall interactions on the suspension behavior, these interactions were not taken into account (the introduction of diffusion terms that 
account for these effects is a work in progress). In the simulations a parabolic velocity profile and an isotropic orientation distribution were prescribed on the inflow boundary and a natural flow-out condition on the exit of a parallel plate channel. The development of the flow kinematics and the evolution of 3-D fiber orientation were analyzed.

\subsubsection{Numerical solution procedure}

The coupled flow kinematics and 3-D fiber orientation distribution were simulated to study the development of 2-D flows of suspensions with continuously-dispersed fibers in a Newtonian fluid through a parallel plate channel. Detailed description of the governing equations, statistical scheme and solution procedure can be found for numerical simulation of multi-disperse fiber suspension in Chiba and Chinesta [49] that we will briefly summarize in this paragraph.

Mass and momentum conservation equations govern the motion of an incompressible material, and the continuum theory developed by Lipscomb et al. [1] was used as a rheological equation. Furthermore, the evolution of $\boldsymbol{p}$, the unit vector defining the fiber axis, in a Newtonian homogeneous flow can be described by the Jeffery equation [27].

The second-order orientation tensor $\boldsymbol{a}_{2}$ (see Eq.(1), $\psi(\boldsymbol{p})$ is the orientation distribution function) introduced by Advani and Tucker [36] is a suitable and concise way of describing the orientation distribution of fibers. The eigenvalues and the eigenvectors of $\boldsymbol{a}_{2}$ give the lengths and directions of the three major axes of the orientation ellipsoid (e.g., Fig. 2). The eigenvalues indicate the intensity of fiber orientation along the corresponding directions (eigenvectors).

$$
\boldsymbol{a}_{2}=\oint \boldsymbol{p} \otimes \boldsymbol{p} \psi(\boldsymbol{p}) d \boldsymbol{p}
$$

We used a statistical scheme in the computations of fiber orientation distribution which proceeds by evaluating the 3$\mathrm{D}$ orientations of a large number of fibers with specified initial angles by solving the Jeffery equation along the streamlines. In the next paragraph we will show the predictions of the streamlines, the evolution of the orientation ellipsoids and the stress distribution for monodispersed suspensions with aspect-ratio $r_{a}=10$ and quasi infinite aspect-ratio fibers as well as almost continuouslydispersed aspect-ratio fibers including $r_{a}=10,12,14, \ldots, 46$, 48 and 50 whose volume fraction is the same.

\subsubsection{Flow kinematics and fiber orientation}

Figures 1(a) to (c) show the development of the streamlines at the Reynolds number $\mathrm{Re}=10$ for three cases.

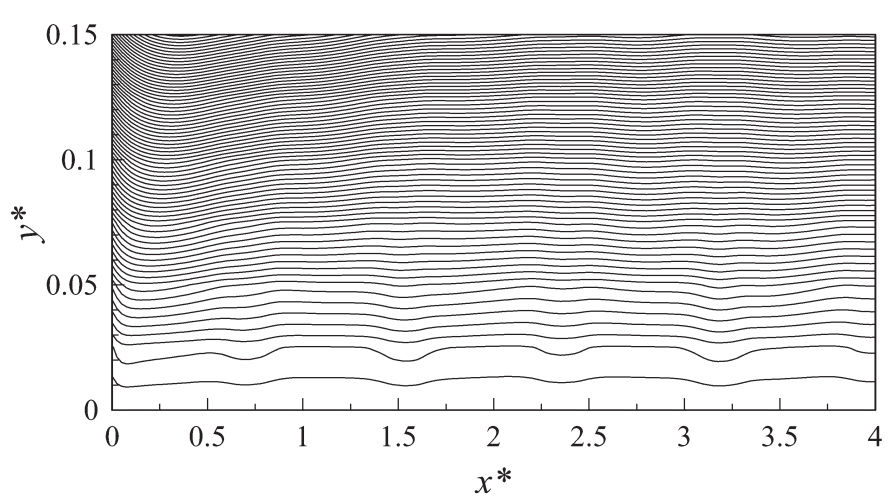

(a) $r_{a}=10$ mono-dispersion

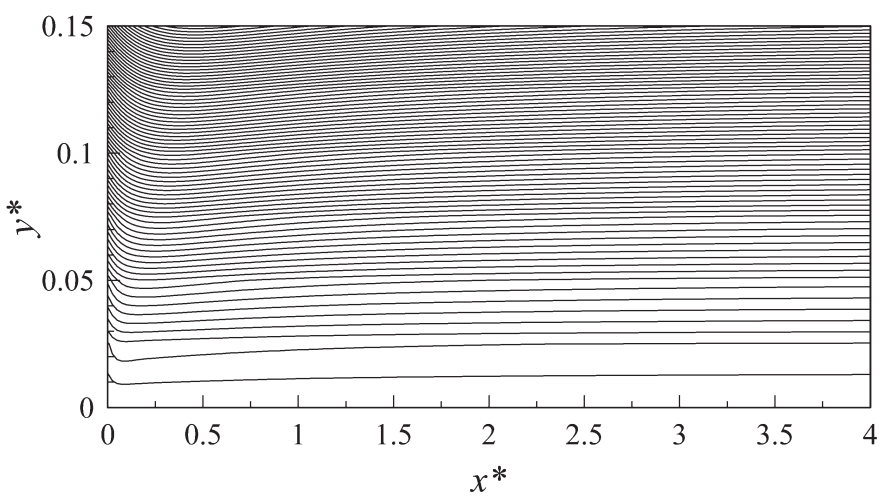

(b) continuously-dispersed aspect-ratio suspension

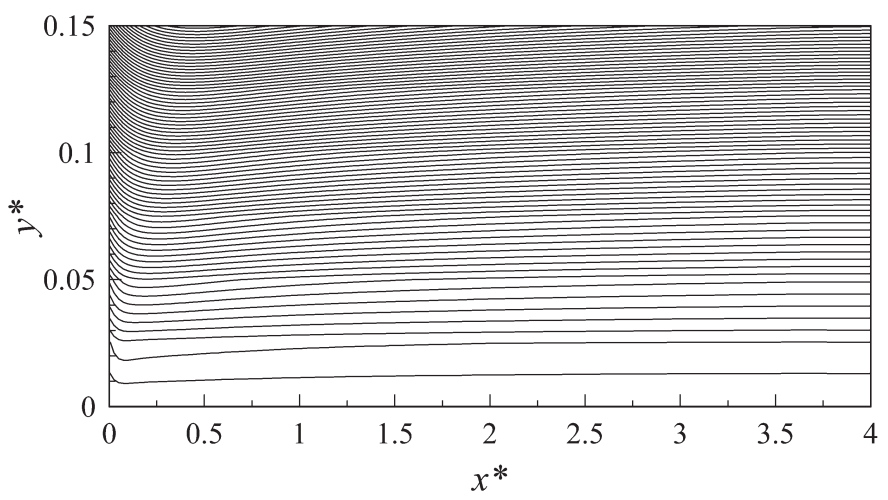

(c) quasi infinite aspect-ratio mono-dispersion

Fig. 1 Development of streamlines in a developing flow of fiber suspension at $\mathrm{Re}=10$. Flow direction is from left to right.

It is found that the streamlines depict smooth curves for quasi infinite aspect-ratio case, while the streamlines become significantly wavy near the channel wall for $r_{a}=10$ mono-disperse suspension and they change into smooth curves as one approaches the centerline. The wavy patterns of the streamlines near the channel wall disappear with an increase in the contribution of large aspect-ratio fibers as shown in the continuously-dispersed aspect-ratio case. These wavy patterns of the streamlines for suspensions with small aspect-ratio fibers arise from the flip-over behavior of individual fibers similarly to the cause of wavy profiles of the centerline velocity (not illustrated in this article, see [67]). 
Figure 2 shows the evolution of the orientation ellipsoids (projections of the ellipsoids on the $x-y$ plane) along the streamlines near the channel wall. For quasi infinite aspectratio case (see Fig. 2(c)), fiber orientation distribution changes from isotropic distribution (drawn by a circle) at the channel inlet to co-linear alignment (all individual fibers align to the streamlines) in the downstream region. The evolution of the orientation ellipsoids is almost similar to the quasi infinite aspect-ratio case for the continuouslydispersed aspect-ratio suspension (see Fig. 2(b)). On the other hand, the orientation ellipsoids frequently and periodically oscillate along the streamlines for suspensions including small aspect-ratio fibers (see Fig. 2(a)), and also the oscillation phenomenon becomes significant as one approaches the channel wall because of large velocity gradient. It is also found in comparison of Fig. 2(a) with

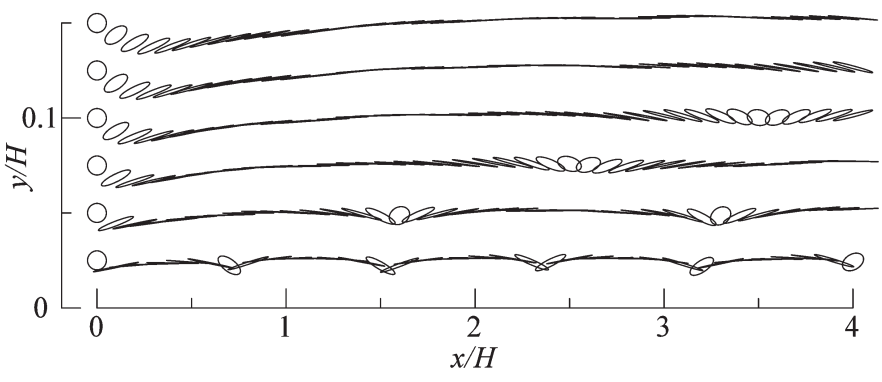

(a) $r_{a}=10$ mono-dispersion

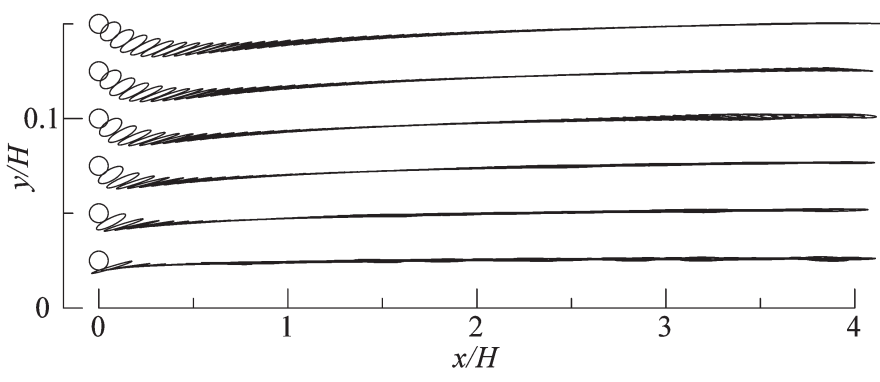

(b) continuously-dispersed aspect-ratio suspension

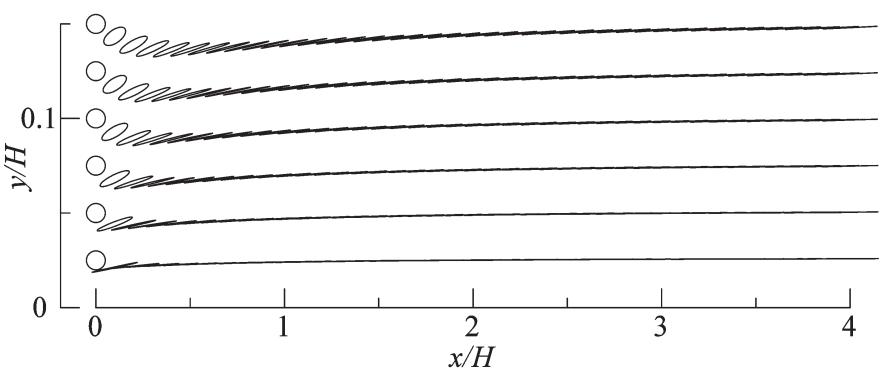

(c) quasi infinite aspect-ratio mono-dispersion

Fig. 2 Evolution of the orientation ellipsoids (projections of the ellipsoids on the flow plane) along the streamlines in a developing flow of fiber suspension at $R e=10$. Flow direction is from left to right. (from [49])
Fig. 2(b) that the period of oscillation phenomenon for multi-disperse suspensions completely depends on the period for the mono-dispersion involving the fiber with the smallest aspect-ratio ( $r_{a}=10$ in this simulation). Finally, the projections of the ellipsoids on the $x-z$ plane (not shown here, see [67]) clearly show that the preferred direction of the orientation ellipsoids always lies on the $x-y$ plane even at the occurrence of oscillation phenomenon owing to the isotropic orientation specified at the channel inlet.

Furthermore, Figs. 3(a) to (c) show that the extremely complex distribution of the principal stress difference, which is observed for the mono-disperse suspension of $r_{a}=10$, is reduced as the number of aspect-ratios increases. For the continuously-dispersed aspect-ratio case, the distribution becomes very smooth and is close to the counterpart of the quasi infinite aspect-ratio mono-disperse case, because the intensity of flip-over or oscillation phenomenon can be suppressed and the orientation ellipsoid exhibits better alignment to the streamline as explained above.

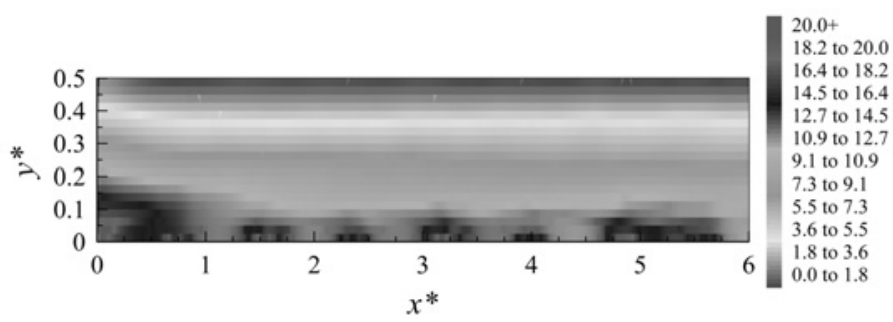

(a) $r_{a}=10$ mono-dispersion

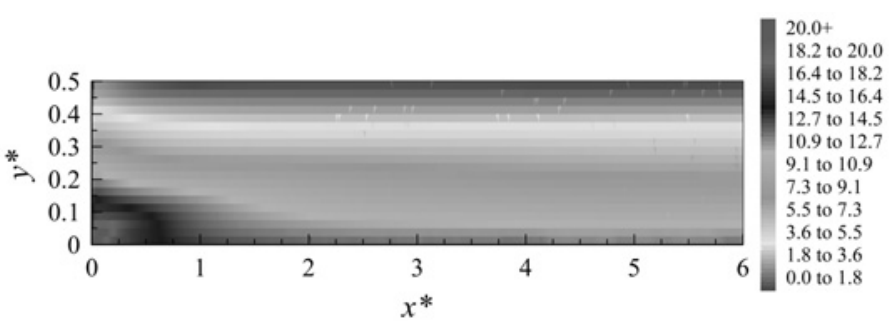

(b) continuously-dispersed aspect-ratio suspension

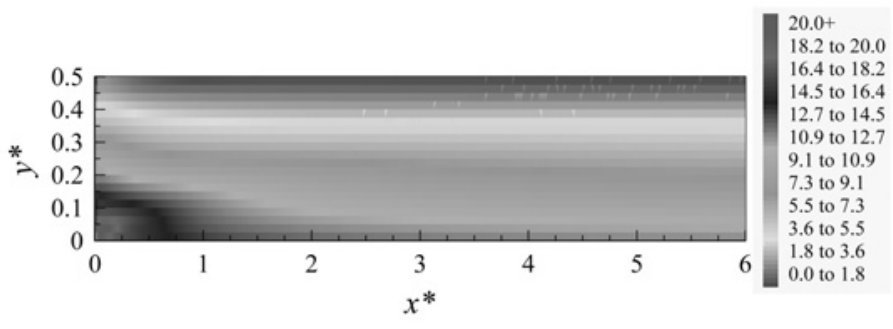

(c) quasi infinite aspect-ratio mono-dispersion

Fig. 3 Principal stress difference in a developing flow of fiber suspension at $\mathrm{Re}=10$. Flow direction is from left to right. (from [67]) 


\subsubsection{Conclusions}

As the number of aspect-ratios included in multi-disperse fiber suspensions increases, the fiber assembly almost completely aligns along the streamline, thus the velocity profile and the stress distribution become smooth, i.e., the wavy patterns of the velocity profile and streamlines as well as the localized and complex variation of stresses near the channel wall are removed. Consequently, the mechanical and physical properties of fiber reinforced composites can be improved by increasing number of aspect-ratios included in the suspension as well as volume fraction related to large fiber aspect-ratios.

\section{2 Orientation of disk-like particles in a micro- composite processing}

\subsubsection{Numerical solution for orientation of thin disk-like particle}

Observation of disk-like particle orientation was conducted in a slit flow as is illustrated in Fig. 4. A slit channel was mounted on the sidewall of a large reservoir, thus the thin disk-like particles were subjected by a planar extensional flow in a reservoir, then by a simple shear flow through a slit channel. The evolution of the 3-D orientation of disk-like particle in both a planar extensional flow and a simple shear flow of Newtonian fluid was simulated by decoupling flow kinematics with particle orientation. The decoupling scheme is largely different from the coupling scheme for the above simulation of fiber suspension case.

The 3-D orientation of a disk-like particle in a 2-D flow parallel to the $x-y$ plane is shown in Fig. 5. The evolution equation of the unit normal $\boldsymbol{p}$, the orientation vector, defining the orientation of a disk-like particle immersed in a Newtonian homogeneous flow can be described by the

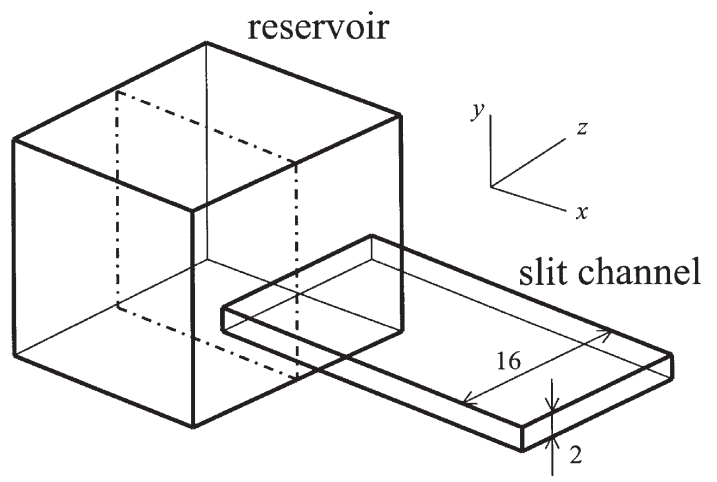

Fig. 4 Schematic diagram of the reservior and slit channel used in the experiment of talc suspension flow.

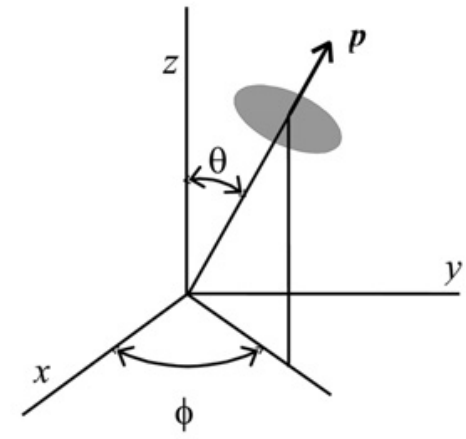

Fig. 5 Orientation of a disk particle and coordinate system.

Jeffery equation similarly to the equation for a slender particle such as a fiber.

\section{(a) in a planar extensional flow}

Figure 6 shows the evolution of the disk-like particle orientation: the projection of the disk-like particles on the $x-y$ plane is illustrated in this figure and a short line drawn from the disk center expresses the orientation vector. It is clearly seen that the orientation vector gradually aligns in the $y$-direction, i.e., we can notice that thin disk-like particles take the parallel orientation to the upper- and lower-walls of the slit channel in a planar extensional flow through the reservoir in the orientation observation experiment.

\section{(b) in a simple shear flow}

Figure 7 shows the orientation evolution of the disk-like particle using the projections of the particles on both the $x-y$ plane and the $x-z$ plane in order to clearly understand the evolution of the 3-D particle orientation. A grey-filled circle expresses a back-face of the particle. We can easily find periodic flip-over of a thin disk-like particle. The period of the shear strain for the flip-over is well known as $\dot{\gamma} T=2 \pi\left(r_{a}+1 / r_{a}\right)$, e.g., $\dot{\gamma} T=63.5$ for $r_{a}=0.1$. Furthermore, for large initial angle $\theta_{0}$ the disk-like particle can be observed as a circle from the $y$-direction in a simple shear flow except the occurrence of flip-over (see Fig. 7(b)). Thin disk-like particles, therefore, keep the parallel orientation to the upper- and lower-walls of the slit channel. This tendency becomes marked as the aspect-ratio is further decreased (see [68]).

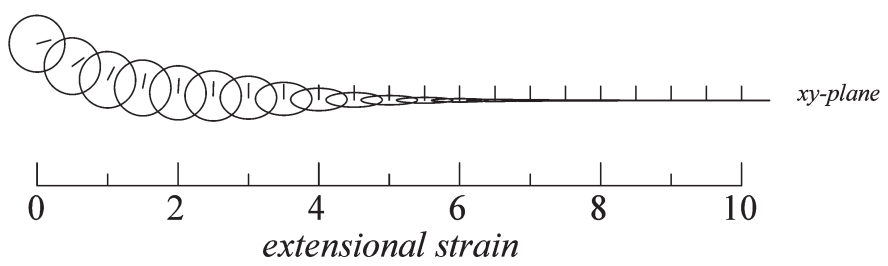

Fig. 6 Evolution of disk-like particle orientation in a planar extensional flow for stretch rate $\dot{\varepsilon}=1 \mathrm{~s}^{-1}$, aspect ratio $r_{a}=0.1$, initial angles $\phi_{0}=15^{\circ}, \theta_{0}=10^{\circ}$. 


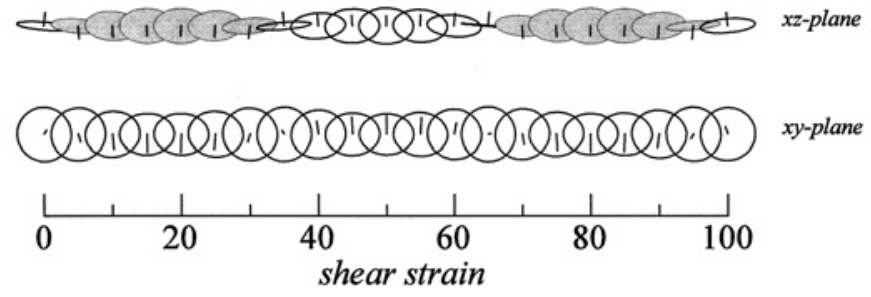

(a) initial angles $\phi_{0}=60^{\circ}, \theta_{0}=10^{\circ}$

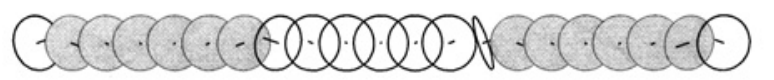

$x z$-plane

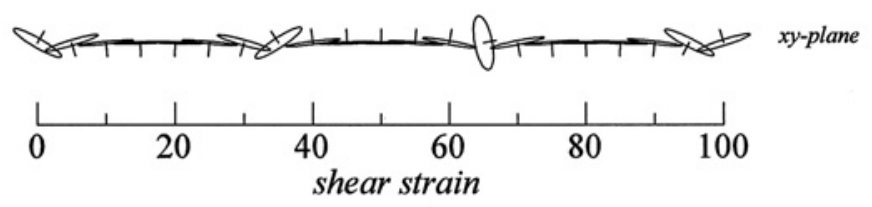

(b) initial angles $\phi_{0}=60^{\circ}, \theta_{0}=80^{\circ}$

Fig. 7 Evolution of disk-like particle orientation in a simple shear flow for shear rate $\dot{\gamma}=5 \mathrm{~s}^{-1}$, aspect ratio $r_{a}=0.1$.

\subsubsection{Observation of the evolution of talc orientation in a slit flow}

$0.01 \mathrm{wt} \%$ talc suspension in glycerin was used in the orientation observations. The size of talc particles is very dispersed from $5 \mu \mathrm{m}$ to $30 \mu \mathrm{m}$ and various shapes can be observed from nearly a circle to a polygon. The photographic images of the talc particle orientations were recorded by a digital video camera attached on a microscope from the upper side of the slit.

Figure 8 shows the evolution of the orientation of talc

particles whose shape is nearly a circle (Fig. 8(a)) and a complex shape (Fig. 8(b)) during a half period of flip-over. The talc particles keep the parallel orientation to the upperand lower-walls of the slit channel except the occurrence of flip-over similarly to the predictions in Fig. 7. Dependence of the flip-over period on the particle shape is recognized from Fig. 8. Furthermore, we can find that the flip-over period is almost the same as each other even for largely different particle size. These phenomena arise from the effect of complex particle shape on rotation of the talc particle and no accurate data of talc thickness: talc thickness is not constant and depends on the talc size, however, it is not possible to measure the thickness of the observed talc particle.

\subsubsection{Conclusions}

In a flow through a slit channel, thin disk-like particles, such as talc and mica particles, keep the parallel orientation to the upper- and lower-walls of the slit channel except occurrence of periodic flip-over. Furthermore, it was confirmed from the migration measurement using talc particle suspension that migration of thin micro particles does not occur in a slit channel flow, thus the concentration distribution is almost uniform (see [69]). We were able to obtain the knowledge of the sheet processing of thin microparticle reinforced composites from the numerical simulations, and observations of the migration and the orientation of thin disk-like particles.

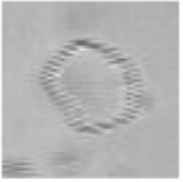

$5.0(\mathrm{~s})$

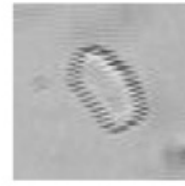

$10.2(\mathrm{~s})$

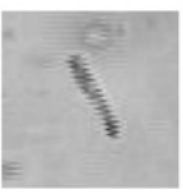

$10.6(\mathrm{~s})$ (a) $0(\mathrm{~s})$

$0.4(s)$

$0.8(\mathrm{~s})$

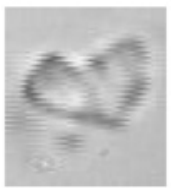

$1.0(\mathrm{~s})$

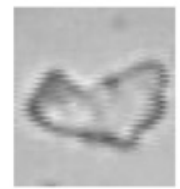

$6.0(\mathrm{~s})$

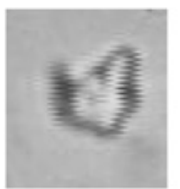

$12.3(\mathrm{~s})$

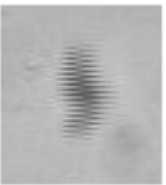

$12.8(\mathrm{~s})$

Fig. 8 Observation of the evolution of talc particle orientation through a slit channel for (a) nearly circular shaped particle, (b) particle with complex geometry. 


\section{Concluding remarks}

The evolution of macro- or micro-structure of suspended particles in suspension flows and flow kinematics induced by the particle structures are so much significant to obtain the knowledge of the processing operations of particle reinforced composites as well as to improve the mechanical and physical properties of the advanced composites. It is a long way, however, to reach this destination.

As reported in the Sec. 3, in a dilute regime of fiber suspensions, the fiber assembly almost completely aligns along the streamline, thus the velocity profile and the stress distribution become smooth as the number of aspect-ratios included in the suspension increases, i.e., the wavy patterns of the velocity profile and streamlines as well as the localized and complex variation of stresses near the channel wall are removed. Then we can conclude that the mechanical and physical properties of composites should be improved by increasing number of fiber aspect-ratios as well as volume fraction related to large fiber aspect-ratios.

In the practical processing of particle reinforced composites, however, the suspension regime corresponds to a semi-concentrated or concentrated regime, thus the Jeffery equation is no more an appropriate way to describe the particle orientation because it does not take account of the particle-particle interaction. This effect can be introduced in the Jeffery formularization from an appropriate Brownian term (including diffusion effects [70]). However, it is not fully understood, thus this question will be addressed in a near future work.

Furthermore, a still open problem that is encountered in the processing of particle-reinforced plastics is the modeling of the role of the interaction between particles and polymer molecules on the evolution of the particle orientation, i.e., an extension of the Jeffery equation to viscoelastic media.

\section{References}

[1] Lipscomb GG, Denn MM, Hur DH, Boger DV (1988) J NonNewtonian Fluid Mech, 26, 297-325

[2] Chiba K, Nakamura K, Boger DV (1990) J Non-Newtonian Fluid Mech, 35, 1-14

[3] Rosenberg J, Denn MM, Keunings R (1990) J Non-Newtonian Fluid Mech, 37, 317-345

[4] Ausias G, Agassant JF, Vincent M, Lafleur PG, Lavoie PA, Carreau PJ (1992) J Rheol, 36, 525-542

[5] Ranganathan S, Advani SG (1993) J Non-Newtonian Fluid Mech, 47, 107-136

[6] Ahmed A, Alexandrou AN (1994) J Non-Newtonian Fluid Mech, 55, 115-136

[7] Altan MC, Rao BN (1995) J Rheol, 39, 581-599
[8] Chung ST, Kwon TH (1995) Polym Eng Sci, 35, 604-618

[9] Baloch A, Webster MF (1995) Comput Fluids, 24, 135-151

[10] Ghosh T, Grmela M, Carreau PJ (1995) Polym Compos, 16, $144-153$

[11] Oyanagi Y, Yamaguchi Y, Kitagawa M, Terao K, Mochizuki M (1981) Kobunshi Ronbunshu, 38, 285-290

[12] Pipes RB, McCullough RL, Taggart DG (1982) Polym Compos, 3, 34-39

[13] Maiti SN, Sharma KK (1992) J Mater Sci, 27, 4605-4613

[14] Karrad S, Lopez Curesta JM, Crespy A (1998) J Mater Sci, 33, 453-461

[15] McInerney LF, Kao N, Bhattacharya SN (2003) Polym Eng Sci, 43, 1821-1829

[16] Nogai T (1982) Sen-i Gakkaishi, 38, T413-T418

[17] White JL, Knutsson BA (1982) Polym Eng Rev, 2, 71-82

[18] Martinsson J, White JL (1986) Polym Compos, 7, 302-314

[19] Lim SH, White JL (1990) J Rheol, 34, 343-366

[20] Papanastasiou TC, Alexandrou AN (1987) J Non-Newtonian Fluid Mech, 25, 313-328

[21] Dinh SM, Armstrong RC (1984) J Rheol, 28, 207-227

[22] Ericksen JL (1960) Kolloid Z, 173, 117-122

[23] Phan-Thien N, Graham AL (1990) Rheol Acta, 29, 433-441

[24] Phan-Thien N, Graham AL (1991) Rheol Acta, 30, 44-57

[25] Shaqfeh ESG, Fredrickson GH (1990) Phys Fluids A, 2, 7-24

[26] Chiba K, Nakamura K (1998) J Non-Newtonian Fluid Mech, 78, 167-185

[27] Jeffery GB (1922) Proc R Soc, A102, 161-179

[28] Chang RY, Shiao FC, Yang WL (1994) J Non-Newtonian Fluid Mech, 55, 1-20

[29] Azaiez J, Guenette R, Ait-Kadi A (1997) J Non-Newtonian Fluid Mech, 73, 289-316

[30] Poitou A, Chinesta F, Torres R (2000) J Non-Newtonian Fluid Mech, 90, 65-80

[31] Chinesta F, Chaidron G, Poitou A (2003) J Non-Newtonian Fluid Mech, 113, 97-125

[32] Akbar S, Altan MC (1992) Polym Eng Sci, 32, 810-822

[33] Hinch EJ, Leal LG (1976) J Fluid Mech, 76, 187-208

[34] Doi M (1981) J Polym Sci, Polym Phys Ed, 19, 229-243

[35] Grmela M, Carreau PJ (1987) J Non-Newtonian Fluid Mech, 23, 271-294

[36] Advani SG, Tucker III CL (1987) J Rheol, 31, 751-784

[37] Advani SG, Tucker III CL (1990) J Rheol, 34, 367-386

[38] Altan MC, Advani SG, Güceri SI, Pipes RB (1989) J Rheol, 33, 1129-1155

[39] Cintra JS, Tucker III CL (1995) J Rheol, 39, 1095-1122

[40] Chung DH, Kwon TH (2002) J Rheol, 46, 169-194

[41] Parsheh M, Brown ML, Aidun CK (2006) J Non-Newtonian Fluid Mech, 136, 38-49

[42] Ericsson KA, Toll S, Manson JAE (1997) J Rheol, 41, 491-511

[43] Chiba K, Nakamura K (1995) Nihon Reoroji Gakkaishi, 23, 159-165 
[44] Altan MC, Güceri SI, Pipes RB (1992) J Non-Newtonian Fluid Mech, 42, 65-83

[45] Chono S, Makino M (1995) Trans Japan Soc Mech Eng Ser B, 61, 3190-3196

[46] Lipscomb GG (1986) Ph.D. dissertation, University of California, Berkeley

[47] Ausias G, Agassant JF, Vincent M (1994) Int Polym Process, 9, 51-59

[48] Chiba K, Chinesta F (2002) Revue des composites et des matériaux avancés, 12, 499-513

[49] Chiba K, Chinesta F (2005) Rheol Acta, 45, 1-13

[50] Kabanemi KK, Hetu JF, Garcia-Rejon A (1997) Int Polym Process, 12, 182-191

[51] Doraiswamy D, Metzner AB (1986) Rheol Acta, 25, 580-587

[52] Zirnsak MA, Boger DV (1998) J Non-Newtonian Fluid Mech, 79, 105-136

[53] Fan XJ, Phan-Thien N, Zheng R (1999) J Non-Newtonian Fluid Mech, 84, 257-274

[54] Öttinger HC (1996) "Stochastic processes in polymeric fluids", Springer, Berlin Heidelberg New York

[55] Milliken WJ, Gottlieb M, Graham AL, Mondy LA, Powell RL (1989) J Fluid Mech, 202, 217-232

[56] Chung DH, Kwon TH (2002) J Non-Newtonian Fluid Mech, 107, 67-96

[57] Schmid CF, Switzer LH, Klingenberg DJ (2000) J Rheol, 44,
781-809

[58] Thomasset J, Grmela M, Carreau PJ (1997) J Non-Newtonian Fluid Mech, 73, 195-203

[59] Yamamoto S, Matsuoka T (1993) J Chem Phys, 98, 644-650

[60] Yamamoto S, Matsuoka T (1996) Polym Eng Sci, 36, 23962403

[61] Joung CG, Phan-Thien N, Fan XJ (2001) J. Non-Newtonian Fluid Mech, 99, 1-36

[62] Joung CG, Phan-Thien N, Fan XJ (2002) J Non-Newtonian Fluid Mech, 102, 1-17

[63] Yamamoto S, Matsuoka T (1997) J Chem Phys, 107, 33003308

[64] Pozrikidis C (2005) Eur J Mech B: Fluids, 24, 125-136

[65] Ausias G, Fan XJ, Tanner RI (2006) J Non-Newtonian Fluid Mech, 135, 46-57

[66] Shiraishi Y, Narazaki N, Kikutani T (2004) Polym Compos, 25, 194-213

[67] Chiba K, Chinesta F (2006) Rheol Acta, 45, 547-554

[68] Yasuda K, Ohara N, Mori N, Chiba K (2005) J Text Mach Soc Japan, 58, T29-T36

[69] Yasuda K, Nishimura M, Chiba K (2006) J Text Eng, 52, 165-169

[70] Chiba K, Ammar A, Chinesta F (2005) Rheol Acta, 44, 406-417 